\title{
Ophthalmic Findings in Systemic Sclerosis and Its Association with Dermal Fibrosis
}

\author{
Janeth Villegas', Bety Yáñez \\ ${ }^{1}$ Rheumatology Department, Dos de Mayo Hospital, Lima, Peru \\ ${ }^{2}$ Ophthalmology Department, Dos de Mayo Hospital, Lima, Peru \\ Email: byanez@hotmail.com
}

How to cite this paper: Villegas, J. and Yáñez, B. (2019) Ophthalmic Findings in Systemic Sclerosis and Its Association with Dermal Fibrosis. Open Access Library Journal, 6: e5672.

https://doi.org/10.4236/oalib.1105672

Received: August 7, 2019

Accepted: September 6, 2019

Published: September 9, 2019

Copyright $\odot 2019$ by author(s) and Open Access Library Inc.

This work is licensed under the Creative Commons Attribution International License (CC BY 4.0).

http://creativecommons.org/licenses/by/4.0/

(c) () Open Access

\begin{abstract}
Objective: To determine frequency and type of ophthalmologic findings in Systemic Sclerosis (SSc) and to establish their association with the extent and severity of cutaneous fibrosis. Methods: Descriptive-transversal study of 34 patients, (January-December 2017). It included patients with limited cutaneous Systemic Sclerosis (lcSS) and diffuse cutaneous Systemic Sclerosis (dcSS). The severity of cutaneous fibrosis was measured using the modified Rodnan skin score (mRSS). The ophthalmologic examination consisted of the evaluation of the palpebral fibrosis and the ocular surface by Schirmer's test, tear rupture time (BUT), OSDI (Ocular Disease Surface Index) and SANDE (Symptom Assessment in Dry Eye) questionnaires, eye fundus and retinography. We use simple logistic regression (OR) to establish the association with $\mathrm{p}<0.05$. Results: 21 patients $(61.7 \%)$ with dcSS and 13 patients $(38.2 \%)$ with lcSS. Women 32 (94.1\%). Mean disease time 67.4 months. The mRSS was mild in $10(29.4 \%)$ and moderate-severe in 24 (70.6\%). The main findings: palpebral fibrosis 30 (88.2\%), keratoconjunctivitis sicca (KCS) 26 (76.5\%), blepharophimosis 20 (58.8\%) and enophthalmos 7 (20.6\%) had alteration of the retinal pigment epithelium $3(8.8 \%)$. Associated with the extension: blepharophimosis, enophthalmos and KCS predominated in dcSS with Schirmer test positive in $52.4 \%$, mild dry eye highlighted in both groups. We found significant association between blepharophimosis and moderate-severe mRSS. Conclusion: Eyelids skin thickening and KCS were more prevalent in dcSS. Moderate-severe mRSS was associated with atrophic changes in the eyelids.
\end{abstract}

\section{Subject Areas}

Ophthalmology 


\section{Keywords}

Systemic Sclerosis, Modified Rodnan Skin Score, Ophthalmic Findings, Ocular Surface Disease, Dry Eye

\section{Introduction}

Systemic sclerosis (SSc) is a chronic disabling disorder characterised by three pivotal aspects, obliterative and proliferative microvascular involvement, activation of the immune system and increase of extracellular matrix deposition in the skin and internal organs [1].

There are two major types of SSc, limited systemic sclerosis (lcSSc) and diffuse systemic sclerosis (dcSSc). Fibrosis is the most characteristic pathological hallmark of SSc, and it is especially prominent in dcSSc.

The modified Rodnan skin score (mRSS), remains the best method for the objective assessment of skin involvement both in clinical practice and in research settings that combines feasibility, an acceptable reliability, responsiveness to change and correlation with physician global correlates of health status [1].

This score is a recognized instrument, which measures skin involvement, palpating the skin, useful to determine the severity and prognosis of the disease.

Ocular complications in SSc have been documented in report of individual cases or small series because it is a rare disease [2]. The most frequently findings are those of the anterior segment, with keratoconjunctivitis sicca (KCS) being the most frequent $37 \%-79 \%$, eyelid alterations produced by the stiffness and tension of the skin, thinner corneas, besides chronic and normal pressure glaucoma [3] [4] [5] [6] [7]. Changes in corneal hysteresis have also been investigated through the use of the Ocular Response Analyzer (ORA) in SSc [8].

In the posterior segment the most described are the retinal microvascular anomalies arguing whether they should be by themselves an SSc or by chronic HTA complication [9].

The objective of the study is to determine the association between the extension and the degree of severity of the cutaneous fibrosis with the ophthalmologic findings using the mRSS in this group of patients.

\section{Materials and Methods}

Descriptive-transversal study of 34 patients attended in the Rheumatology Service (January-December 2017). It included patients with dcSSc and lcSSc. Subjects were selected non-systematically taking into account the inclusion criteria: age $\geq 15$ years (informed consent signed by tutor), compliance with SSc classification criteria ACR/EULAR 2013. Exclusion criteria were, diabetes mellitus prior to diagnosis of SSc, HIV, pregnancy, is sine SSc, overlapping syndromes, undifferentiated connective tissue disease, severe or malignant HTA.

The severity of cutaneous fibrosis was measured using mRSS, performed ac- 
cording to previously described parameters by a single rheumatologist, to avoid biases [10]. The ophthalmologist was blind with respect to the clinical subtype of the patient with SSc.

Data were collected in two files. In rheumatology file, demographic, clinical variables and mRSS obtained values were recorded. In ophthalmology file, symptoms and ocular antecedents were noted. Visual acuity was taken with better correction. Ocular surface examination consisted of biomicroscopy with slit lamp, measurement of tear rupture time (BUT), Schirmer's test with anesthesia (Optitech Eyecare, Inc., U.P., India), fluoresceine staining and Goldmann tonometry, OSDI (Ocular Disease Surface Index; Allergan, Inc, Irvine, CA) [11] and SANDE (Symptom Assessment in Dry Eye) [12] questionnaires, also eye fundus and retinography were done. The study was approved by the hospital Ethics committee.

The statistical analysis was performed using the SPSS-IBM program (Version 22). Continuous variables were expressed as means and standard deviation, while categorical variables such as frequencies and percentages. Parametric (Student's T) and non-parametric (Mann-Whitney U) tests were used to compare continuous variables according to data distribution. Fisher's exact test was used to compare categorical variables. Statistical analysis and correlation between the results of the questionnaires were evaluated using the Spearman correlation coefficient and the clinical differences were determined using the Bland-Altman analysis. A value of P 0.05 was considered significant.

\section{Results}

We evaluated $21(67.7 \%)$ patients with dcSS and 13 (38.2\%) with lcSSc. There were 32 women $(94.1 \%)$. The mean age was 51.18 years (range $28-86$ ) and mean illness time was 67.4 months. The mRSS was mild in 10 (29.4\%) and moderate-severe in $24(70.6 \%)$. Table 1 summarizes demographic data and general characteristics of the patients.

The most frequent symptom was ocular dryness 29 (85.3\%). The ocular surface disease was the most prevalent finding, characterized by palpebral fibrosis $30(88.2 \%)$, KCS 26 (76.5\%), blepharophimosis 20 (58.8\%) and enophthalmos 7 (20.6\%). In posterior segment, Table 2 shows that the most prevalent finding was the alteration of the retinal pigment epithelium $3(8.8 \%)$.

The mean of the final values of the questionnaires was: OSDI, $28.2 \pm 18.65$ and $28.3 \pm 18.39$ for dcSSc and lcSS respectively and Sande, $39.5 \pm 27.85$ and $29.4 \pm 25.77$ for dcSSc and lcSSc respectively. The scores obtained with the two questionnaires were significantly correlated $(\mathrm{R}=0.445, \mathrm{P}<0.001)$. The Altman Bland analysis showed a lesser difference of two grad units between the scores of the two questionnaires.

Table 3 shows the ophthalmological findings versus SSc type. We found statistically significant association between blepharophimosis and dcSS $p=0.00$, OR 7.2 $(1.5,33.8)$. The results showed a major frequency of positive BUT so 
Table 1. Demographic data and general characteristics of the patients.

\begin{tabular}{cc}
\hline Characteristics & Patients \\
\cline { 2 - 2 } Age (years) & $\mathrm{N}=34$ \\
mean & 51.18 \\
range & $28-86$ \\
SD & 14.3 \\
Sex n, \% & \\
female & $32 / 94.11$ \\
male & $2 / 5.89$ \\
Time of disease (months) & \\
mean & 67.41 \\
range & $3-300$ \\
SD & 72.09 \\
HTA n, \% & $2 / 8.8$ \\
Systemic Sclerosis (SSc) & \\
diffuse (dcSSc) & $21 / 61.76$ \\
limited (lcSSc) & $13 / 38.2$ \\
mild & $14 / 41.2$ \\
moderate-severe & $20 / 58.8$ \\
\hline Modified Rodnan skin score n, \% &
\end{tabular}

Table 2. Ocular symptoms and findings.

\begin{tabular}{ccc}
\hline & N & $\%$ \\
\hline Symptoms & 3 & 8.8 \\
Red eye & 6 & 17.6 \\
Low vision & 29 & 85.3 \\
Dry eye & 13 & 38.2 \\
Use of lubricant eye drops & 4 & 11.8 \\
Ocular surgery & & \\
Ocular disease & 2 & 5.9 \\
Glaucoma & 2 & 5.9 \\
Macular degeneration & 1 & 2.9 \\
Uveitis & & \\
Anterior segment findings & 30 & 88.2 \\
Palpebral skin thickening & 26 & 76.5 \\
Keratoconjunctivitis sicca & 20 & 58.8 \\
Blepharophimosis & 7 & 20.6 \\
Enophtalmos & 2 & 5.9 \\
Lagophtalmos & & 2.9 \\
Posterior segment findings & 3 & \\
Alteration of the retinal pigment epithelium & & \\
HTA Retinopathy & & \\
\hline
\end{tabular}


Table 3. Ocular findings vs systemic sclerosis type.

\begin{tabular}{|c|c|c|c|c|c|c|}
\hline & \multicolumn{6}{|c|}{ Type of Systemic sclerosis } \\
\hline & \multicolumn{2}{|c|}{ Limited } & \multicolumn{2}{|c|}{ Difuse } & \multirow{2}{*}{$\mathbf{P}$} & \multirow{2}{*}{ OR IC } \\
\hline & $\mathrm{N}$ & $\%$ & $\mathrm{~N}$ & $\%$ & & \\
\hline $\begin{array}{l}\text { Palpebral skin thickening } \\
\text { (moderate-severe) }\end{array}$ & 8 & 61.5 & 16 & 70.6 & 0.36 & $0.5(0.1,2.2)$ \\
\hline Enophtalmos & 1 & 7.7 & 6 & 28.6 & 0.14 & $4.8(0.5,44.5)$ \\
\hline Blepharophimosis & 4 & 30.8 & 16 & 76.2 & 0.09 & $7.2(1.5,33.8)$ \\
\hline Blepharitis (moderate-severe) & 1 & 7.7 & 4 & 19.1 & 0.36 & $0.3(0.0,3.5)$ \\
\hline Keratoconjunctivitis sicca & 16 & 76.2 & 10 & 76.9 & 0.96 & $0.9(0.1,4.9)$ \\
\hline \multicolumn{7}{|l|}{ Schirmer Test with anesthesia } \\
\hline Positive & 4 & 30.8 & 11 & 52.4 & 0.26 & $2.4(0.5,10.6)$ \\
\hline Negative & 9 & 69.2 & 10 & 47.6 & & \\
\hline \multicolumn{7}{|l|}{ BUT } \\
\hline Positive & 11 & 84.6 & 20 & 95.2 & 0.41 & $3.6(0.2,44.7)$ \\
\hline Negative & 2 & 15.4 & 1 & 4.8 & & \\
\hline \multicolumn{7}{|l|}{ OSDI } \\
\hline Normal-mild & 11 & 78.6 & 17 & 85.0 & 0.62 & $1.5(0.2,9.0)$ \\
\hline Severe & 3 & 21.4 & 3 & 15.0 & & \\
\hline \multicolumn{7}{|l|}{ SANDE } \\
\hline Normal-mild & 10 & 71.4 & 15 & 75.0 & 0.8 & $1.2(0.2,5.5)$ \\
\hline Severe & 4 & 28.6 & 5 & 25.0 & & \\
\hline
\end{tabular}

much in dcSSc and lcSSc the same without being observed with the Schirmer test.

Table 4 presents the results when comparing ophthalmologic findings and mRSS. A statistically significant association was found between blepharophimosis $\mathrm{p}=0.00$, OR $20(3.5,122.0)$ and enophthalmos $\mathrm{p}=0.01$, OR $2.0(1.4,3.0)$ with moderate-severe mRSS. High values of BUT positive were obtained in the two categories of mRSS, moderate to severe $95 \%$ and mild $85.7 \%$.

\section{Discussion}

Systemic sclerosis, is an immune-mediated rheumatic chronic disease that is characterised by fibrosis of the skin and internal organs and vasculopathy [13].

Skin thickening is a universal feature of SSc. Skin thickness in SSc is caused by increased collagen, intercellular matrix formation in the dermis and oedema, which is probably caused by both microvascular injury and inflammation. Because of the accumulation of collagen and fluid, the skin thickens, making it impossible to pinch it into a normal skin fold. At the following stage the skin becomes shiny, taut and adherent to the subcutis [14]. 
Table 4. Ocular findings vs modified Rodnan skin score.

\begin{tabular}{|c|c|c|c|c|c|c|}
\hline & \multicolumn{6}{|c|}{ Modified Rodnan skin score } \\
\hline & \multicolumn{2}{|c|}{ Mild } & \multicolumn{2}{|c|}{ Moderate-Severe } & \multirow{2}{*}{$\mathbf{P}$} & \multirow{2}{*}{ OR IC } \\
\hline & $\mathbf{N}$ & $\%$ & $\mathrm{~N}$ & $\%$ & & \\
\hline $\begin{array}{l}\text { Palpebral skin thickening } \\
\text { (moderate-severe) }\end{array}$ & 8 & 61.5 & 16 & 70.6 & 0.22 & $0.4(0.08,1.8)$ \\
\hline Enophtalmos & 1 & 7.7 & 6 & 28.6 & 0.74 & $6.4(0.6,61.1)$ \\
\hline Blepharophimosis & 4 & 30.8 & 16 & 76.2 & 0.00 & $34(4.9,235.6)$ \\
\hline Blepharitis (moderate-severe) & 1 & 7.7 & 4 & 19.1 & 0.35 & $2.4(0.3,17.0)$ \\
\hline Keratoconjunctivitis sicca & 11 & 78.6 & 15 & 75.0 & 0.80 & $0.8(0.1,4.1)$ \\
\hline \multicolumn{7}{|l|}{ Schirmer test with anesthesia } \\
\hline Positive & 4 & 30.8 & 11 & 52.4 & 0.26 & $2.2(0.5,9.0)$ \\
\hline Negative & 9 & 69.2 & 10 & 47.6 & & \\
\hline \multicolumn{7}{|l|}{ BUT } \\
\hline Positive & 11 & 84.6 & 20 & 95.2 & 0.41 & $2.7(0.2,33.8)$ \\
\hline Negative & 2 & 15.4 & 1 & 4.8 & & \\
\hline \multicolumn{7}{|l|}{ OSDI } \\
\hline Normal-mild & 8 & 61.5 & 9 & 42.9 & 0.62 & $1.5(0.2,9.0)$ \\
\hline Severe & 3 & 23.1 & 8 & 38.1 & & \\
\hline \multicolumn{7}{|l|}{ SANDE } \\
\hline Normal-mild & 10 & 71.4 & 15 & 75.0 & 0.8 & $1.2(0.2,5.5)$ \\
\hline Severe & 4 & 28.6 & 5 & 25.0 & & \\
\hline
\end{tabular}

The mRSS is a validated instrument that evaluates cutaneous thickening. It is done by palpating the skin. It is useful to determine the severity and prognosis of the disease. High values of this score are associated with poor prognosis of the disease and lower survival. It can have as disadvantages, the lack of objectivity and the bias between examiners, for this reason this exam was carried out by a single examiner in this study [10] [15] [16].

Our results are consistent with previous studies in which the ocular surface alteration was the most prevalent finding, palpebral fibrosis $88.2 \%$ and KCS 76.5\%. There are published case-control reports that evaluate the association between clinical signs and dry eye symptoms in SSc. Waszczykowska A et al. [5], found $22 \%$ of KCS and increased intraocular pressure $\mathrm{p}<0.002$. In Latin America, Gomes B et al. [4], in a descriptive study of 45 patients in Brazil, found abnormalities in the skin of the eyelids $48.9 \%$ and KCS $42.2 \%$; Uribe C [6], in Colombia, in dcSSc patients found that they had 5.25 times higher risk of presenting primary open angle glaucoma. In this study two patients (5.9\%) had been diagnosed with glaucoma.

Rentka et al. [17] found a significant correlation between the OSDI question- 
naire and the duration of SSc. The data obtained in our study showed a good correlation between the OSDI and SANDE questionnaires, evidencing that both can be used to support the diagnosis of dry eye in SSc patients. Similar to Gomes et al. [18] and Rentka et al. [17] our results not demonstrated a association between subjective and objective dry eye parameters in these cohort of SSc patients that could be explained by the idiosyncratic nature of the patients or the decrease in the corneal sensation that occurs in the dry eye.

Only one recent study, from Ayhan et al. [19], analyzes the association between skin score with corneal biomechanics and dry eye tests in SSc, finding a statistical correlation between mRSS and the Schirmer test in dcSSc and with the OSDI questionnaire in dcSSc and lcSSc. Our results showed no statistically significant association with these dry eye tests but with clinical changes in the eyelids, blepharophimosis $\mathrm{p}=0.00$, OR $20(3.5,122.0)$ and enophthalmos $\mathrm{p}=0.01$, OR $2.0(1.4,3.0)$ with mRSS moderate-severe. These are associated with a greater degree of skin fibrosis and frequently occur in advanced stages of the disease.

Fibrosis caused by SSc in the eyelids skin will trigger a decrease in blinking and finally lead to an alteration in the production of tears and the secretion of the meibomian glands producing a dry eye.

In the posterior segment, the most frequent finding was the alteration of the retinal pigment epithelium 3 (8.8\%). Case-control studies are inconclusive about the changes observed in retina, unlike the choroidal vasculature appears to be more affected than the retinal microcirculation [20].

Although the ocular complications of SSc are not the most frequent or the most serious, knowing them will help to diagnose and treat them early and will improve the "limited" quality of life of patients suffering from this systemic chronic disease.

\section{Conclusion}

Our results are consistent with previous studies in which the ocular surface alteration was the most prevalent finding, palpebral fibrosis $88.2 \%$ and KCS $76.5 \%$. Also we found a statistically significant association with clinical changes in the eyelids, blepharophimosis $\mathrm{p}=0.00$, OR $20(3.5,122.0)$ and enophthalmos $\mathrm{p}=$ 0.01 , OR $2.0(1.4,3.0)$ with mRSS moderate-severe. These changes are associated with a greater degree of skin fibrosis and frequently occur in advanced stages of the disease.

\section{Acknowledgements}

The authors wish to acknowledge the assistance provided by Norma Pletikosic Silva M. D. in revising the text and Sixto Sánchez Calderón M.D. for his support with the statistical analysis.

\section{Conflicts of Interest}

The authors declare no conflicts of interest regarding the publication of this paper. 


\section{References}

[1] Barsotti, S., Stagnaro, C. and Della Rossa, A. (2015) Systemic Slerosis: A Critical Digest of the Recent Literature. Clinical Experimental Rheumatology, 33, S3-S14.

[2] Tailor, R., Gupta, A., Herrick, A. and Kwartz, J. (2008) Ocular Manifestations of Scleroderma. Survey of Ophthalmology, 54, 292-304.

https://doi.org/10.1016/j.survophthal.2008.12.007

[3] West, R.H. and Barnett, A.J. (1979) Ocular Involvement in Scleroderma. British Journal of Ophthalmology, 63, 845-847. https://doi.org/10.1136/bjo.63.12.845

[4] Gomes, B.A.F., Santhiago, M.R., Magalhäes, P., Kara-Junior, N., Azevedo, M.N.L., et al. (2011) Ocular Findings in Patients with Systemic Sclerosis. Clinics, 66, 379-385. https://doi.org/10.1590/S1807-59322011000300003

[5] Waszczykowska, A., Gos, R., Waszczykowska, E., Dziankowska-Bartkowiak, B. and Jurowski, P. (2013) Prevalence of Ocular Manifestations in Systemic Sclerosis Patients. Archives of Medical Science, 9, 1107-1113. https://doi.org/10.5114/aoms.2013.39217

[6] Uribe Laiseca, C.M. (2012) Manifestaciones oculares de esclerosis sistémica: Estudio de casos y controles. Tesis Universidad de Colombia, Facultad de Medicina, Bogotá. http://www.bdigital.unal.edu.co/11351/1/5598736.2012.pdf

[7] Perez, M.C. (2015) Esclerosis Sistémica en pacientes colombianos. Experiencia de un centro y revisión de la literatura. Tesis, Universidad CES, Antioquia. http://hdl.handle.net/10946/4225

[8] Emre, S., Kayikcjoglu, O., Ates, H., Cinar, E., Inceoglu, N., et al. (2010) Corneal Hysteresis, Corneal Resistance Factor, and Intraocular Pressure Measurements in Patients with Scleroderma Using the Reichert Ocular Response Analyzer. Cornea, 29, 628-631. https://doi.org/10.1097/ICO.0b013e3181c3306a

[9] Ushiyama, O., Ushiyama, K., Yamada, T., Koarada, S., Tada, Y., et al. (2003) Retinal Findings in Systemic Sclerosis: A Comparison with Nailfold Capillaroscopic Patterns. Annals of the Rheumatic Diseases, 62, 204-207. https://doi.org/10.1136/ard.62.3.204

[10] Rodnan, G.P., Lipinski, E. and Luksick, J. (1979) Skin Thickness and Collagen Content in Progressive Systemic Sclerosis and Localized Scleroderma. Arthritis \& Rheumatology, 22, 130-140. https://doi.org/10.1002/art.1780220205

[11] Schiffman, R.M., Christianson, M.D., Jacobsen, G., Hirsch, J.D. and Reis, B.L. (2000) Reliability and Validity of the Ocular Surface Disease Index. Archives of Ophthalmology, 118, 615-621. https://doi.org/10.1001/archopht.118.5.615

[12] Gulati, A., Sullivan, R., Buring, J.E., Sullivan, D.A. and Schaumberg, D.A. (2006) Validation and Repeatability of a Short Questionnaire for Dry Eye Syndrome. American Journal of Ophthalmology, 142, 125-131. https://doi.org/10.1016/j.ajo.2006.02.038

[13] Denton, C.P. and Khanna, D. (2017) Systemic Sclerosis. The Lancet, 390, 1685-1699. https://doi.org/10.1016/S0140-6736(17)30933-9

[14] Varga, J. and Abraham, D. (2007) Systemic Sclerosis: A Prototypic Multisystem Fibrotic Disorder. Journal of Clinical Investigation, 117, 557-567. https://doi.org/10.1172/JCI31139

[15] Czirjak, L., Foeldvari, I. and Muller-Ladner, U. (2008) Skin Involvement in Systemic Sclerosis. Rheumatology, 47, 44-45. https://doi.org/10.1093/rheumatology/ken309

[16] Furst, D.E., Clements, P.J., Steen, V.D., Medsper, T.A., Masi, A.T., et al. (1998) The Modified Rodnan Skin Score Is an Accurate Reflection of Skin Biopsy Thickness in 
Systemic Sclerosis. The Journal of Rheumatology, 25, 84-88.

[17] Rentka, A., Nagy, A., Harsfalvi, J., Szucs, G., Szekanecz, Z., et al. (2017) Association between Objective Signs and Subjective Symptoms of Dry Eye Disease in Patients with Systemic Sclerosis. Rheumatology International, 37, 1835-1845.

https://doi.org/10.1007/s00296-017-3794-2

[18] Gomes, B.A.F., Santhiago, M.R., de Azevedo, M.N. and Moraes, H.V. (2012) Evaluation of Dry Eye Signs and Symptoms in Patients with Systemic Sclerosis. Graefe's Archive for Clinical and Experimental Ophthalmology, 250, 1051-1056. https://doi.org/10.1007/s00417-012-1938-3

[19] Ayhan, Z., Kaya, M., Ozturk, T., Arikan, G. and Birlik, M. (2018) Association between Skin Thickness Measurements with Corneal Biomechanical Properties and Dry Eye Tests in Systemic Sclerosis. Ocular Immunology and Inflammation, 1-6. https://doi.org/10.1080/09273948.2018.1501496

[20] Kreps, E.O., Carton, C., Cutolo, M, Cutolo, C.A., Vanhaecke, A., et al. (2019) Ocular Involvement in Systemic Sclerosis: A Systemic Literature Review, It's Not All Scleroderma That Meets the Eye. Seminars in Arthritis and Rheumatism, 49, 119-125. https://doi.org/10.1016/j.semarthrit.2018.12.007 